\title{
INVARIANCE OF THE POINCARÉ NUMBERS OF A DISCRETE GROUP
}

\author{
BY OSWALD VEBLEN
}

Some time ago Professor J. Nielsen of Copenhagen kindly pointed out to me that the last statement in $\S 30$, page 142 , of my Colloquium Lectures on Analysis Situs is incorrect; i. e., it is not true that "(12) is a necessary condition that (10) shall be a transformation to a new set of generators of G." For in solving equation (11) account must be taken of the generating relations.

This error invalidates the argument given in the next two sections for the invariance of the Poincare numbers of a group $G$. It therefore seems worth while to point out the following rather obvious way of proving this invariance.

We are considering a group $G$ determined by a finite number of generators

$$
g_{1}, g_{2}, \ldots, g_{n}
$$

and a finite number of generating relations

$$
\begin{gathered}
g_{1}^{a_{i 1}} g_{2}^{a_{i}} \cdots g_{n}^{a_{\text {in }}} g_{1}^{b_{i 1}} g_{2}^{b_{i 2}} \cdots g_{n}^{b_{i n}} \cdots g_{1}^{j_{i 1}} g_{2}^{j_{12}} \cdots g_{n}^{j_{\text {in }}}=1 \\
(i=1,2, \ldots, k) .
\end{gathered}
$$

This group determines uniquely a commutative group $\tilde{G}$ defined by the condition that it has $n$ generators subject to (1) and the condition that all operations of the group be commutative. In view of the commutativity, the generating relations of $\tilde{G}$ reduce to

where

$$
\begin{gathered}
g^{\gamma_{i 1}} g^{\gamma_{i 2}} \cdots g^{\gamma_{i n}}=1 \\
(i=1,2, \ldots, k)
\end{gathered}
$$

$$
\gamma_{i t}=a_{i t}+b_{i t}+\cdots+j_{i t} .
$$

For the group $\tilde{G}$, a new set of generators $h_{1}, h_{2}, \ldots, h_{n}$ can be found, as explained in $\S 31$, for which the generating 
relations are

$$
h_{1}^{d_{1}}=1, \quad h_{2}^{d_{2}}=1, \quad \ldots, \quad h_{r}^{d_{r}}=1
$$

where those of the exponents $d_{1}, d_{2}, \ldots, d_{r}$ which are not 1 are the invariant factors of the matrix $\left|\gamma_{i t}\right|$.

An operation of $\tilde{G}$ is of finite period if and only if it is in the group $H$ generated by the operations $h_{1}, h_{2}, \ldots, h_{r}$ and the relations (3). Hence $H$ contains all operations of $\tilde{G}$ of finite period. Moreover $H$ is a finite group because it is commutative and generated by a finite number of operations each of finite period. The invariant factors of $\left|\gamma_{i t}\right|$ are invariants of $H$ (Kronecker, BerLINER MONATsBerichte, 1870, p. 885).

These invariant factors are what Tietze calls the Poincaré numbers of the group $G$. They are invariants of $G$ because $G$ determines the commutative group $\tilde{G}$ uniquely and $\tilde{G}$ determines the finite group $H$ uniquely and $H$ determines the invariant factors uniquely.

Princeton University

\section{ANALYTIC FUNCTIONS AND PERIODICITY*}

BY J. F. RITT

This paper presents two theorems which show that the condition that a function be periodic can be analyzed, in different ways, into a set of requirements, from the satisfaction of only one of which, if the function is analytic, the periodicity of the function can be inferred. The theorems are

THEOREM A. If $f(z)$ is a uniform analytic function, and if an $a>0$ exists such that every $z_{1}$ at which $f(z)$ is analytic is the center of a circle of radius a on which a $z_{2}$ lies at which $f(z)$ is analytic and assumes the same value as at $z_{1}$, then $f(z)$ is periodic, and has a period of modulus a.

* Presented to the Society May 3, 1924. 